\title{
ABUNDANCES OF DEUTERIUM AND HELIUM-3 IN THE PROTOSOLAR CLOUD
}

\author{
J. GEISS \\ International Space Science Institute, Hallerstrasse 6, 3012 Bern, Switzerland \\ G. GLOECKLER \\ Department of Physics, University of Maryland, College Park MD 20742, USA, and Department of \\ Atmospheric, Oceanic and Space Sciences, University of Michigan, Ann Arbor, MI 48109, USA
}

\begin{abstract}
The mass spectrometric determinations of the isotopic composition of helium in the solar wind obtained from (1) the Apollo Solar Wind Composition (SWC) experiment, (2) the Ion Composition Instrument (ICI) on the International Sun Earth Explorer 3 (ISEE-3), and (3) the Solar Wind Composition Spectrometer (SWICS) on Ulysses are reviewed and discussed, including new data given by Gloeckler and Geiss (1998). Averages of the ${ }^{3} \mathrm{He} /{ }^{4} \mathrm{He}$ ratio in the slow wind and in fast streams are given. Taking account of separation and fractionation processes in the corona and chromosphere, ${ }^{3} \mathrm{He} /{ }^{4} \mathrm{He}=(3.8 \pm 0.5) \times 10^{-4}$ is derived as the best estimate for the present-day Outer Convective Zone (OCZ) of the sun. After corrections of this ratio for secular changes caused by diffusion, mixing and ${ }^{3} \mathrm{He}$ production by incomplete H-burning (Vauclair, 1998), we obtain (D + $\left.{ }^{3} \mathrm{He}\right) / \mathrm{H}=(3.6 \pm 0.5) \times 10^{-5}$ for the Protosolar Cloud (PSC). Adopting ${ }^{3} \mathrm{He} / \mathrm{H}=(1.5 \pm 0.2) \times 10^{-5}$ for the PSC, as is indicated from the ${ }^{3} \mathrm{He} /{ }^{4} \mathrm{He}$ ratio in the 'planetary gas component' of meteorites and in Jupiter (Mahaffy et al., 1998), we obtain $(\mathrm{D} / \mathrm{H})_{\text {protosolar }}=(2.1 \pm 0.5) \times 10^{-5}$. Galactic evolution studies (Tosi, 1998) show that the measured D and ${ }^{3} \mathrm{He}$ abundances in the Protosolar Cloud and the Local Interstellar Cloud (Linsky, 1998; Gloeckler and Geiss, 1998), lead to $(\mathrm{D} / \mathrm{H})_{\text {primordial }}=$ $(2-5) \times 10^{-5}$. This range corresponds to a universal baryon/photon ratio of $(6.0 \pm 0.8) \times 10^{-10}$, and to $\Omega_{\mathrm{b}}=0.075 \pm 0.015$.
\end{abstract}

\section{Introduction}

So far, primordial abundances of deuterium and helium-3 are deduced from observations and measurements in three samples of cosmic material. (1) The present-day galaxy, (2) the Protosolar Cloud (PSC), and (3) clouds with very low contamination from stellar nucleosynthesis, using metallicity as an indicator. In this paper, we present estimates for the abundances of D and ${ }^{3} \mathrm{He}$ in the Protosolar Cloud that represents a sample of galactic material with a nucleosynthetic age of approximately $4.6 \mathrm{~Gy}$. Since D was converted into ${ }^{3} \mathrm{He}$ in the early sun, the abundance of ${ }^{3} \mathrm{He}$ in the Outer Convective Zone (OCZ) of the sun corresponds approximately to the abundance of D $+{ }^{3} \mathrm{He}$ in the PSC. We summarise and review the ${ }^{3} \mathrm{He} /{ }^{4} \mathrm{He}$ abundance measurements in the solar wind, from which we then obtain an estimate of the ${ }^{3} \mathrm{He} /{ }^{4} \mathrm{He}$ ratio in the present-day OCZ. After a discussion of the processes that have changed the $\mathrm{He} / \mathrm{H}$ and ${ }^{3} \mathrm{He} /{ }^{4} \mathrm{He}$ ratios in the $\mathrm{OCZ}$ over the lifetime of the sun, we give our best estimate for $\left(\mathrm{D}+{ }^{3} \mathrm{He}\right) / \mathrm{H}$ in the PSC. The protosolar $\mathrm{D} / \mathrm{H}$ ratio is then obtained by combining the solar wind ${ }^{3} \mathrm{He} /{ }^{4} \mathrm{He}$ data with the ${ }^{3} \mathrm{He} /{ }^{4} \mathrm{He}$ ratio in the PSC that is estimated from the values in the 'planetary gas component' of meteorites and in Jupiter. Finally, by comparing the D and ${ }^{3} \mathrm{He}$ abundances in 
the PSC and in the Local Interstellar Cloud (LIC) we discuss constraints on the evolution of these two nuclei in the galaxy and on their primordial abundances.

\section{The ${ }^{3} \mathrm{He} /{ }^{4} \mathrm{He}$ Ratio in the Solar Wind}

The ${ }^{3} \mathrm{He} /{ }^{4} \mathrm{He}$ ratio in the solar wind has been measured by several spaceborne instruments. Since the results clearly show that this ratio is not constant, a comprehensive database and at least some understanding of the causes for the changes in ${ }^{3} \mathrm{He} /{ }^{4} \mathrm{He}$ are needed for obtaining the best estimate for the ${ }^{3} \mathrm{He} /{ }^{4} \mathrm{He}$ ratio in the present-day OCZ. Comprehensive results have been obtained from three investigations: (1) the Apollo Solar Wind Composition (SWC) experiments, using solar wind collection in foils with subsequent analysis by laboratory mass spectrometry, (2) the Ion Composition Instrument (ICI) on the International Sun Earth Explorer 3 (ISEE-3) using an electromagnetic mass spectrometer allowing unambiguous measurement of the mass/charge ratio of the ions, and (3) the Solar Wind Ion Composition Spectrometer (SWICS) on Ulysses, a time-of-flight system giving the mass/charge ratio as well as the mass of the ions. The results obtained with these three techniques are given in Table I. True variations in the ${ }^{3} \mathrm{He} /{ }^{4} \mathrm{He}$ ratio were found by all three experiments. However, for comparable solar wind flows (cf. the averages in the slow wind) the agreement between the results obtained over 25 years by these three completely different techniques is very remarkable. Data not explicitly given in Table I include those obtained by electrostatic analysers (Bame et al., 1968; Grünwaldt, 1976) and a result from a collection experiment flown with a Luna mission (Boltenkov et al., 1972). Some of these data represent special events, emphasizing the variability in the ${ }^{3} \mathrm{He} /{ }^{4} \mathrm{He}$ ratio, but they do not indicate a disagreement with the more general results given in Table I.

Prior to the Ulysses mission, all ${ }^{3} \mathrm{He} /{ }^{4} \mathrm{He}$ data were taken in the ecliptic plane, where the low speed solar wind dominates, although some data were obtained in fast streams and during CME events. The SWC-Apollo foils collected solar wind particles mainly during slow wind conditions. Since the technique employed by this experiment gave very small systematic errors, we assign a high weight to the SWC-Apollo results in calculating the average ${ }^{3} \mathrm{He} /{ }^{4} \mathrm{He}$ ratio in the slow wind from the Apollo, ISEE-3 and Ulysses data. Thanks to the polar orbit of the spacecraft and the large energy range of the instrument, SWICS-Ulysses has allowed the first systematic investigation of the helium isotopes in the high speed streams coming out of large coronal holes. The result is that ${ }^{3} \mathrm{He} /{ }^{4} \mathrm{He}$ is lower in the fast streams than it is in the average slow solar wind. The uncertainties in the absolute ${ }^{3} \mathrm{He} /{ }^{4} \mathrm{He}$ ratios obtained in the fast streams and in the slow wind are larger than the uncertainties in the difference. We have taken this into account in our estimate for the average of ${ }^{3} \mathrm{He} /{ }^{4} \mathrm{He}$ in fast streams. As Table I shows, Bodmer et al. (1995) reported a smaller difference than Gloeckler and Geiss (1998). In part, this is due to a different choice of detector combinations in the SWICS instrument. 
Table I

Results of mass spectrometric measurements of ${ }^{3} \mathrm{He} /{ }^{4} \mathrm{He}$ in the solar wind.

\begin{tabular}{cll}
\hline & $\begin{array}{l}{ }^{3} \mathrm{He} /{ }^{4} \mathrm{He} \\
{\left[10^{-4}\right]}\end{array}$ & \\
\hline SWC Apollo 11-16 (1969-1972) & & \\
Weighted average of 5 missions & $4.26 \pm 0.21$ & $\mathrm{a}$ \\
Average over flux and time & $4.36 \pm 0.25$ & $\mathrm{a}$ \\
Long-time average & & \\
Average of ratios & $4.88 \pm 0.48$ & $\mathrm{~b}, \mathrm{c}, \mathrm{d}$ \\
Slow Wind $(V<500 \mathrm{~km} / \mathrm{s})$ & $4.37 \pm 0.5$ & $\mathrm{~d}$ \\
Slow Wind $(V<500 \mathrm{~km} / \mathrm{s})$ & $4.8 \pm 0.48$ & $\mathrm{c}, \mathrm{d}$ \\
Fast Stream $(V>700 \mathrm{~km} / \mathrm{s})$ & & \\
Slow Wind $(V<500 \mathrm{~km} / \mathrm{s})$ & $4.6 \pm 0.6$ & $\mathrm{e}$ \\
Fast Stream $(V>700 \mathrm{~km} / \mathrm{s})$ & $4.08 \pm 0.6$ & $\mathrm{e}$ \\
SWICS Ulysses $(1991-1996)$ & $3.3 \pm 0.3$ & $\mathrm{f}$ \\
Slow Wind, Adopted Average & $4.3 \pm 0.4$ & \\
Fast Stream, Adopted Average & $3.6 \pm 0.5$ &
\end{tabular}

(a) Geiss et al., 1970a; Geiss et al., 1972;

(b) Ogilvie et al., 1980;

(c) Coplan et al., 1984;

(d) Bochsler, 1984; the error for the average of ratios was adopted from the error of the long-time average;

(e) Bodmer et al., 1995; the given errors include an estimate of the systematic error;

(f) Gloeckler and Geiss, 1998.

Bodmer (1996) has since compared three detector combinations and found ratios between ${ }^{3} \mathrm{He} /{ }^{4} \mathrm{He}$ in the slow wind and in fast streams ranging from 1.07 to 1.25. In determining the ${ }^{3} \mathrm{He} /{ }^{4} \mathrm{He}$ ratio representative for fast streams, we have given more weight to the new data (Gloeckler and Geiss, 1998), because they represent a broader range of solar latitudes than the earlier result of Bodmer et al. (1995).

\section{3. ${ }^{3} \mathrm{He} /{ }^{4} \mathrm{He}$ in the Outer Convective Zone of the Sun}

Elemental and isotopic abundances in the solar wind are not exactly the same as they are in its source reservoir, the Outer Convective Zone (OCZ) of the sun. Separation processes in the chromosphere and in the corona produce a general variability in solar wind ion abundances as well as systematic differences between solar wind and OCZ composition.

In this section, we present two independent derivations of the isotopic composition of helium in the OCZ, the one based on the ${ }^{3} \mathrm{He} /{ }^{4} \mathrm{He}$ ratio in the fast streams, the other on the ${ }^{3} \mathrm{He} /{ }^{4} \mathrm{He}$ ratio in the slow wind. 
Table II

Derivation of the ${ }^{3} \mathrm{He} /{ }^{4} \mathrm{He}$ abundance ratio in the Outer Convective Zone of the sun. Corrections to the observed solar wind ${ }^{3} \mathrm{He} /{ }^{4} \mathrm{He}$ ratios.

\begin{tabular}{|c|c|}
\hline & ${ }^{3} \mathrm{He} /{ }^{4} \mathrm{He}$ Correction \\
\hline \multicolumn{2}{|l|}{ Fast Streams, ${ }^{3} \mathrm{He} /{ }^{4} \mathrm{He}=(3.6 \pm 0.5) \times 10^{-4}$} \\
\hline Chromospheric Separation & $(0 \pm 4) \%$ \\
\hline Corona / Solar Wind Acceleration & \\
\hline \multicolumn{2}{|l|}{ Slow Wind, ${ }^{3} \mathrm{He} /{ }^{4} \mathrm{He}=(4.3 \pm 0.4) \times 10^{-4}$} \\
\hline Chromospheric Separation & $(0 \pm 4) \%$ \\
\hline Corona / Solar Wind Acceleration & $-(8 \pm 5) \%$ \\
\hline \multicolumn{2}{|l|}{ Outer Convective Zone } \\
\hline from Fast Stream Data & ${ }^{3} \mathrm{He} /{ }^{4} \mathrm{He}=(3.6 \pm 0.5) \times 10^{-4}$ \\
\hline from Slow Wind Data & ${ }^{3} \mathrm{He} /{ }^{4} \mathrm{He}=(4.0 \pm 0.5) \times 10^{-4}$ \\
\hline
\end{tabular}

An ion-atom separation process operating in or near the chromosphere causes an overabundance of elements with low first ionisation potential (the "FIP-Effect") and an underabundance of helium in the plasma that is fed into the corona. Specifics concerning the dynamics and geometry of the separation mechanism remain uncertain (cf. Hénoux and Somov, 1992; von Steiger et al., 1997). However, solar flare particle and solar wind data (cf. Garrard and Stone, 1994; von Steiger et al., 1997), covering more than a dozen species with very different atomic properties, indicate that the FIP effect results from a competition between ionisation time in the outer chromosphere and a characteristic ion-atom separation time. This observation allows the fractionations of the ${ }^{3} \mathrm{He} /{ }^{4} \mathrm{He}$ and $\mathrm{He} / \mathrm{H}$ ratios to be linked.

In fast streams, the $\mathrm{He} / \mathrm{H}$ ratio is remarkably constant at 0.05 (Bame et al., 1977). With $\mathrm{He} / \mathrm{H}=0.084$ in the OCZ (Pérez Hernández and Christensen-Dalsgaard, 1994), this gives a depletion of ${ }^{4} \mathrm{He}$ by $40 \%$. If atom-ion separation in the chromosphere is controlled by diffusion, the separation lengths would scale with $m^{1 / 4}$. Since the ionisation times for ${ }^{3} \mathrm{He}$ and ${ }^{4} \mathrm{He}$ are the same (cf. von Steiger and Geiss, 1989), a depletion of ${ }^{3} \mathrm{He}$ by $42 \%$ is calculated, i.e. the chromospheric process would cause a decrease in the ${ }^{3} \mathrm{He} /{ }^{4} \mathrm{He}$ ratio by $\sim 3.5 \%$. This estimate agrees very well with the decrease obtained by the numerical models of von Steiger and Geiss (1989). On the other hand, if the helium depletion in the gas fed into the corona should be caused by some sort of gravitational settling (cf. Barraclough et al., 1996), an increase in ${ }^{3} \mathrm{He} /{ }^{4} \mathrm{He}$ of a similar magnitude could result. We adopt $0 \pm 4 \%$ (cf. Table II) for the ${ }^{3} \mathrm{He} /{ }^{4} \mathrm{He}$ separation in the chromosphere or below.

As compared to the slow wind, the fast streams are more steady and homogenous, variations in elemental abundances and charge states are smaller, and the expansion geometry is fairly well known. Therefore, the fast streams are much better represented by existing steady-state models than the slow wind. Theories and numerical calculations show that much of the kinetic energy in the flow is supplied by wave heating and momentum transfer from waves (Munro and Jackson, 
1977; Bürgi and Geiss, 1986; McKenzie et al., 1997; Banaszkiewicz et al., 1998) which, if non-resonant, gives the same thermal velocity or acceleration to all ion species (Hollweg, 1974; Bürgi and Geiss, 1986). Ion heating by waves is directly supported by the SOHO-UVCS results which show that the ion temperatures in coronal holes are much higher than the electron temperatures, and they increase with increasing mass (Kohl et al., 1997). Iron has the highest mass and mass/charge ratio among the elements monitored in the solar wind, but we have as yet not seen a depletion of the Fe/Mg ratio in the fast streams. Since there is no evidence for ion fractionation in the process of solar wind acceleration out of coronal holes, we assume that the only correction to be applied to the ${ }^{3} \mathrm{He} /{ }^{4} \mathrm{He}$ ratio in fast streams is the chromospheric correction (Table II).

$\mathrm{The} \mathrm{He} / \mathrm{H}$ ratio in the slow wind is quite variable, daily averages vary typically between 0.035 and 0.05. Observation and theory (cf. Bürgi and Geiss, 1986) indicate that these variations are due to helium settling in the corona and losses in the solar wind acceleration process (see below). The typical upper value of 0.05 for the daily averages of $\mathrm{He} / \mathrm{H}$ indicates that the chromospheric effect on the $\mathrm{He} / \mathrm{H}$ ratio is similar for the fast streams and the slow wind. Therefore, we adopt the $0 \pm 4 \%$ correction also for the slow wind (Table II).

If we have $\mathrm{He} / \mathrm{H}=0.05$ in the plasma coming up from the chromosphere, the depletion below 0.05 must occur in the corona. There, helium is fully ionised, and thus the dynamical behaviour of ${ }^{3} \mathrm{He}^{++}$lies somewhere between $\mathrm{H}^{+}$and ${ }^{4} \mathrm{He}^{++}$. The slow wind represents a mixture of flow types, and since expansion geometries and wave fields are not well known, we do not have an adequate theoretical description. Temporary excursions of the ${ }^{3} \mathrm{He} /{ }^{4} \mathrm{He}$ ratio to high values in the slow wind were observed by several instruments (Bame et al., 1968; Geiss et al., 1970; Grünwaldt, 1976; Coplan et al., 1984; Gloeckler et al., 1997; Gloeckler and Geiss, 1998). Certainly, these high ratios show that, on average, ${ }^{3} \mathrm{He} /{ }^{4} \mathrm{He}$ in the slow wind is enhanced above the OCZ abundance ratio, although there is as yet no adequate theoretical explanation for these excursions. However, even under relatively quiet slow wind conditions some degree of helium isotope fractionation must be expected. Steady-state models give some information on the relative behaviour of ion species, and they show that ${ }^{4} \mathrm{He}^{++}$can be much more depleted than ${ }^{3} \mathrm{He}^{++}$ (Geiss et al., 1970; Bürgi and Geiss, 1986). In the absence of significant momentum transfer from waves, the relative dynamical behaviour of heavy ions in a protonelectron gas is characterised by a drag-factor which in its most simple form is given by $\Gamma=Z^{2} /(2 A-Z-1)$, where $Z$ is the charge state, and $A$ the mass number of the ion species (Geiss et al., 1970). Thus, for ${ }^{3} \mathrm{He}^{++},{ }^{4} \mathrm{He}^{++},{ }^{16} \mathrm{O}^{6+}$ and ${ }^{56} \mathrm{Fe}^{11+}$ the $\Gamma$-factors are $1.33,0.80,1.44$ and 1.21 , respectively. These figures show that ${ }^{4} \mathrm{He}^{++}$is particularly disadvantaged when escape from the solar gravitational field depends strongly on the momentum transfer from the proton-electron gas.

The average ${ }^{4} \mathrm{He} / \mathrm{H}$ ratio in the slow wind is 0.038 , corresponding to a reduction of $\sim 25 \%$ caused by coronal separation processes. Considering the large difference in the drag factors for the two helium isotopes, the depletion of ${ }^{3} \mathrm{He} / \mathrm{H}$ in the 
slow wind could be considerably less. We take this and an occasional, inadvertant admixture of plasma with anomalously high ${ }^{3} \mathrm{He}$ abundance (Gloeckler et al., 1997) into account by assigning a large error to the increase of ${ }^{3} \mathrm{He} /{ }^{4} \mathrm{He}$ in the slow wind, which we adopt to be $(8 \pm 5) \%$ (Table II).

The corrections here lead to the two values for the ${ }^{3} \mathrm{He} /{ }^{4} \mathrm{He}$ ratio in the OCZ given at the bottom of Table II. Giving the two determinations equal weight, we obtain for the Outer Convective Zone

$$
\left({ }^{3} \mathrm{He} /{ }^{4} \mathrm{He}\right)_{\mathrm{OCZ}}=(3.8 \pm 0.5) \times 10^{-4}
$$

We have discussed here in some detail the corrections needed to derive ${ }^{3} \mathrm{He} /{ }^{4} \mathrm{He}$ in the OCZ from the solar wind data. The aim was to show that these corrections can be estimated by straightforward physical arguments, using the observed solar wind $\mathrm{He} / \mathrm{H}$ abundance pattern, independent of very particular assumptions which go into model calculations. We emphasize that the net correction is relatively minor, the average of the ${ }^{3} \mathrm{He} /{ }^{4} \mathrm{He}$ ratios in the fast streams and the slow wind being only $4 \%$ higher than the $\left({ }^{3} \mathrm{He} /{ }^{4} \mathrm{He}\right)_{\mathrm{OCZ}}$ value given above (cf. Tables I and II).

\section{4. $\left(\mathrm{D}+{ }^{3} \mathrm{He}\right) / \mathrm{H}, \mathrm{D} / \mathrm{H}$ and ${ }^{3} \mathrm{He} / \mathrm{H}$ in the Protosolar Cloud}

When 4.6 Gy ago a fragment of an interstellar cloud collapsed to form the solar system, the sun was largely formed by direct infall (Tscharnuter, 1987) implying that the material going into the sun was representative of the Protosolar Cloud. In the early sun, D was converted into ${ }^{3} \mathrm{He}$ which has not been further processed in the material of the Outer Convective Zone (OCZ), as can be surmised from the existence there of beryllium (Geiss and Reeves, 1972). ${ }^{9} \mathrm{Be}$ is indeed much faster destroyed than ${ }^{3} \mathrm{He}$ at all temperatures (Figure 1). Thus, the ${ }^{3} \mathrm{He} /{ }^{4} \mathrm{He}$ ratio in the OCZ basically represents the protosolar $\left(\mathrm{D}+{ }^{3} \mathrm{He}\right) / \mathrm{H}$ ratio. There are, however, two processes that could have changed the ${ }^{3} \mathrm{He} /{ }^{4} \mathrm{He}$ ratio in the Outer Convective Zone during the lifetime of the sun.

Solar seismic data and solar models show that $\mathrm{He} / \mathrm{H}$ in the $\mathrm{OCZ}$ is $16 \%$ lower than it was in the PSC (Pérez Hernández and Christensen-Dalsgaard, 1994; Bahcall and Pinsonneault, 1995). The difference is interpreted as being due to settling of helium out of the OCZ into deeper layers of the sun. ${ }^{3} \mathrm{He}$ settles more slowly than ${ }^{4} \mathrm{He}$, and model calculations (Gautier and Morel, 1997) show an increase of a few percent of the ${ }^{3} \mathrm{He} /{ }^{4} \mathrm{He}$ ratio in the present-day OCZ.

The second possible change of $\left({ }^{3} \mathrm{He} /{ }^{4} \mathrm{He}\right)_{\mathrm{OCZ}}$ over solar history is due to solar mixing. Well below the OCZ, at intermediate levels in the sun, the p-p reaction slowly produces ${ }^{3} \mathrm{He}$, which is not converted into ${ }^{4} \mathrm{He}$, because a much higher temperature would be required (cf. Figure 1). Some of the ${ }^{3} \mathrm{He}$ produced by this incomplete $\mathrm{H}$-burning could have been convected upwards during solar history, thus increasing ${ }^{3} \mathrm{He} /{ }^{4} \mathrm{He}$ in the OCZ (cf. Schatzman and Maeder, 1981; Bochsler et al., 1990). Using mixing models to various solar depths, Vauclair $(1998,1998$ a) 


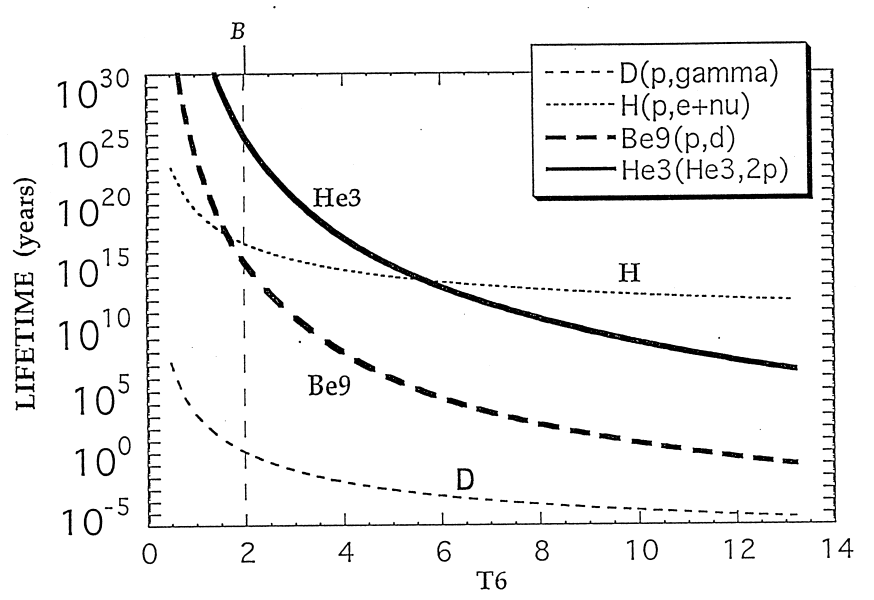

Figure 1. Lifetimes of light nuclei as a function of temperature. Given is the lifetime due to the fastest destructive reaction of the species (cf. insert). Density and composition were normalised to the present conditions at the bottom of the Outer Convective Zone (OCZ). The dashed line (B) is the present temperature at the bottom of the OCZ.

has shown that in order to deplete lithium by two orders of magnitude, as is required, some p-p produced ${ }^{3} \mathrm{He}$ might be added to the OCZ, possibly increasing ${ }^{3} \mathrm{He} /{ }^{4} \mathrm{He}$ by several percent.

On the lunar surface, we have a record of solar wind irradiation that goes back to about $4 \mathrm{~Gy}$. In most materials, the ${ }^{3} \mathrm{He} /{ }^{4} \mathrm{He}$ ratio of the old solar wind samples has been affected by strong diffusive losses of helium. The loss is least severe in ilmenite, and from the results of an on-line etching technique applied to this mineral, Wieler et al. (1992) have deduced that the change in the solar wind ${ }^{3} \mathrm{He} /{ }^{4} \mathrm{He}$ ratio over the last $3 \mathrm{~Gy}$ was less than 10 percent. Since the peak of ${ }^{3} \mathrm{He}$ increases over solar history and moves slowly outward, we expect a large fraction of the contamination of the $\mathrm{OCZ}$ with ${ }^{3} \mathrm{He}$ from incomplete $\mathrm{H}$-burning to have occurred during the last 3 billion years of solar history. Thus settling of helium out of the OCZ and solar mixing could not have increased the ${ }^{3} \mathrm{He} /{ }^{4} \mathrm{He}$ ratio in the OCZ by much more than $10 \%$. We thus adopt a correction of $(5 \pm 3) \%$ and, using $\mathrm{He} / \mathrm{H}=0.10$ for the PSC (Bahcall and Pinsonneault, 1995), we obtain

$$
\left[\left(\mathrm{D}+{ }^{3} \mathrm{He}\right) / \mathrm{H}\right]_{\text {Protosolar }}=(3.6 \pm 0.5) \times 10^{-5}
$$

from the ${ }^{3} \mathrm{He} /{ }^{4} \mathrm{He}$ ratio in the present-day OCZ.

The solar wind data give only the protosolar abundance of the sum of $\mathrm{D}$ and ${ }^{3} \mathrm{He}$. For distinguishing between the two species, an estimate for the ${ }^{3} \mathrm{He} /{ }^{4} \mathrm{He}$ ratio in the PSC is needed. In the past, ${ }^{3} \mathrm{He} /{ }^{4} \mathrm{He}=1.5 \times 10^{-4}$, as measured in the "planetary gas component' of meteorites (Black, 1972; Eberhardt, 1974) was widely used as the best approximation to the protosolar value. In 1996, a Jovian ${ }^{3} \mathrm{He} /{ }^{4} \mathrm{He}$ value of $(1.1 \pm 0.2) \times 10^{-4}$ was obtained by the Galileo Probe Mass Spectrometer (Niemann et al., 1996). This result was somewhat surprising, because one would expect the 
'planetary component' to be depleted, rather then augmented in ${ }^{3} \mathrm{He}$, because most trapping and/or loss mechanisms would tend to decrease the ${ }^{3} \mathrm{He} /{ }^{4} \mathrm{He}$ ratio in the helium that is retained in the solid phase. Since the new Jupiter value (Mahaffy $e t$ al., 1998; Niemann et al., 1998) is higher than the meteoritic value, this puzzle is now resolved, and we adopt here $(1.5 \pm 0.2) \times 10^{-4}$ for the protosolar ${ }^{3} \mathrm{He} /{ }^{4} \mathrm{He}$ ratio, which is compatible with the presently available Jovian and meteoritic data. Thus we obtain from (2)

$$
(\mathrm{D} / \mathrm{H})_{\text {protosolar }}=(2.1 \pm 0.5) \times 10^{-5}
$$

The error limits in (2) and (3) are $1 \sigma$-uncertainties. They include experimental errors, uncertainties in the correction for chromospheric and coronal effects on the solar wind composition, and the uncertainty resulting from helium settling and admixture of p-p produced ${ }^{3} \mathrm{He}$ to the OCZ.

Concerning the best estimate for $(\mathrm{D} / \mathrm{H})_{\text {protosolar }}$, we give in this paper priority to the value derived from the solar ${ }^{3} \mathrm{He} /{ }^{4} \mathrm{He}$ abundance ratio. Determinations of Jovian $\mathrm{D} / \mathrm{H}$ by different methods have given values which disagree by a factor of two and more (cf. Niemann et al., 1996). We note, however, that the $\mathrm{D} / \mathrm{H}$ ratio obtained from ISO infrared spectra of molecular hydrogen $\left(2.2 \times 10^{-5}\right.$; Encrenaz et $a l ., 1996)$ and the new ratio from the Galileo Probe Mass Spectrometer (Mahaffy et al., 1998; Niemann et al., 1998) are close to the (D/H) protosolar value derived here, cf.(3). Furthermore, gas losses from the protoplanetary disc or from the Jovian sub-nebula could in two ways have increased the $\mathrm{D} / \mathrm{H}$ ratio in the planet: the escape of gas under the influence of gravity would (1) favour $\mathrm{H}_{2}$ over DH, and (2) enhance the grain component which is probably enriched in $\mathrm{D}$, leading to an increased $\mathrm{D} / \mathrm{H}$ ratio in the material going into Jupiter.

The method of deriving the protosolar deuterium abundance from the ${ }^{3} \mathrm{He} /{ }^{4} \mathrm{He}$ ratio in the solar wind has been used for twenty-five years (Geiss and Reeves, 1972: $\left.(\mathrm{D} / \mathrm{H})_{\text {protosolar }}=(2.5 \pm 1.0) \times 10^{-5}\right)$. As additional solar wind data $(\mathrm{cf}$. Table II), new estimates of $\mathrm{He} / \mathrm{H}$ in the $\mathrm{OCZ}$ and PSC, other data for the protosolar ${ }^{3} \mathrm{He} /{ }^{4} \mathrm{He}$ ratio, and revised assumptions or model results on solar mixing and fractionation processes in the solar atmosphere became available, several authors have used the same method for deriving $(\mathrm{D} / \mathrm{H})_{\text {protosolar }}$ (Reeves et al., 1973: $(2.6 \pm 1.0) \times 10^{-5}$; Anders and Grevesse, 1989: $(3.4 \pm 0.1) \times 10^{-5}$; Geiss, 1993: $(2.6 \pm 1.0) \times 10^{-5}$; Gautier and Morel, 1997: $\left.(3.01 \pm 0.17) \times 10^{-5}\right)$. All these results, as well as our result of $(2.1 \pm 0.5) \times 10^{-5}$ (cf. 3$)$ lie within the error bars given in 1972, indicating the robustness of the method.

\section{Implications for Galactic Evolution and Cosmology}

With the Protosolar Cloud (PSC) and the Local Interstellar Cloud (LIC) we have two galactic samples, differing in nucleosynthetic age by $4.6 \mathrm{~Gy}$, for which we have reliable data on the isotopic abundances of both hydrogen and helium. $\mathrm{D} / \mathrm{H}$ in the 
LIC (Linsky, 1998) is lower than it was in the PSC. On the other hand, ${ }^{3} \mathrm{He} /{ }^{4} \mathrm{He}$ in the LIC (Gloeckler and Geiss, 1998) is higher than it was in the PSC. The direction of these changes is as expected. Because $\mathrm{D}$ is destroyed but not produced by stars, the $\mathrm{D} / \mathrm{H}$ ratio ought to decrease monotonously with time. ${ }^{3} \mathrm{He}$, on the other hand, is both destroyed and produced by stars. The observed increase in ${ }^{3} \mathrm{He}$ from the PSC to the LIC value is mainly due to p-p production in small stars (cf. Tosi, 1998), a stellar population that began to leave the main sequence and to lose material only relatively late in galactic history.

The LIC is of course not a direct descendent of the PSC. However, since (a) we have good $\mathrm{D}$ and ${ }^{3} \mathrm{He}$ abundance data for both, (b) they evolved at roughly the same distance from the galactic centre and (c) their age difference is not small, but corresponds to $>30 \%$ of the age of the universe, a comparison of the two clouds provides us with unique information on galactic evolution. The PSC is the sample with the best defined nucleosythetic status. Our knowledge on elemental and isotopic abundances in the LIC is still scarce, but will be growing, thanks to refined spectroscopic methods, and to direct measurements on interstellar grains and gas components that pass through the heliosphere. At the present time, the data do not allow a determination of the difference in the $\mathrm{He} / \mathrm{H}$ ratio or in metallicity between LIC and PSC.

Thus, in Figure 2 we plot the abundance ratios as a function of nucleosynthetic age, with the age of the universe taken as $\sim 14$ Gy (cf. Tammann, 1998). Since interstellar deuterium is destroyed and not produced by stars and since production of $\mathrm{D}$ by cosmic rays is minor, the curve labelled "Minimum $\mathrm{D} / \mathrm{H}$ " gives the lower limit of primordial D/H $\left(\sim 2 \times 10^{-5}\right)$. The curve labelled "Steep Decrease D/H" connects the $\mathrm{D} / \mathrm{H}$ ratios in the LIC and the PSC with the primordial deuterium abundance of $\mathrm{D} / \mathrm{H}=5 \times 10^{-5}$ given by Tosi (1998) as the upper bound derived by galactic evolution models. We note that the low $\mathrm{D} / \mathrm{H}$ values obtained in high$z$ clouds given by Burles and Tytler (1998) are consistent with this range of primordial $\mathrm{D} / \mathrm{H}$ ratios. Higher values are difficult to reconcile with the $\mathrm{D} / \mathrm{H}$ and ${ }^{3} \mathrm{He} /{ }^{4} \mathrm{He}$ abundance ratios measured in the PSC and LIC.

From the primordial deuterium abundance given here, $(\mathrm{D} / \mathrm{H})_{\text {primordial }}=(2-$ 5) $\times 10^{-5}$ (cf. Figure 2), the theory of Standard Big Bang Nucleosynthesis (Walker et al., 1991) allows the determination of ranges for other cosmologically relevant parameters: $\left({ }^{3} \mathrm{He} / \mathrm{D}\right)_{\text {primordial }}=0.29-0.54$; the baryon/photon ratio in units of $10^{-10}, \xi_{10}=6.0 \pm 0.8$; the present baryonic density $\rho_{\mathrm{b}, 0}=(4.7-3.6) \times$ $10^{-31} \mathrm{~g} / \mathrm{cm}^{3}$; and (with $H_{0}=55 \pm 7 \mathrm{~km} / \mathrm{s} / \mathrm{Mpc}$, cf. Tammann, 1998) the present relative baryonic density $\Omega_{\mathrm{b}}=0.075 \pm 0.015$. This range of $\Omega_{\mathrm{b}}$ values derived from galactic data is compatible with the lower limit of $\Omega_{b}>0.06$ obtained from Lyman absorption lines (Rauch et al., 1997; Tammann, 1998) and confirms that the baryonic matter density is insufficient to close the universe as has been known for more than two decades (cf. Geiss and Reeves, 1972; Reeves et al., 1973). There is mounting evidence (Tammann, 1998) that the observed total density $\Omega_{\text {tot }}$ 


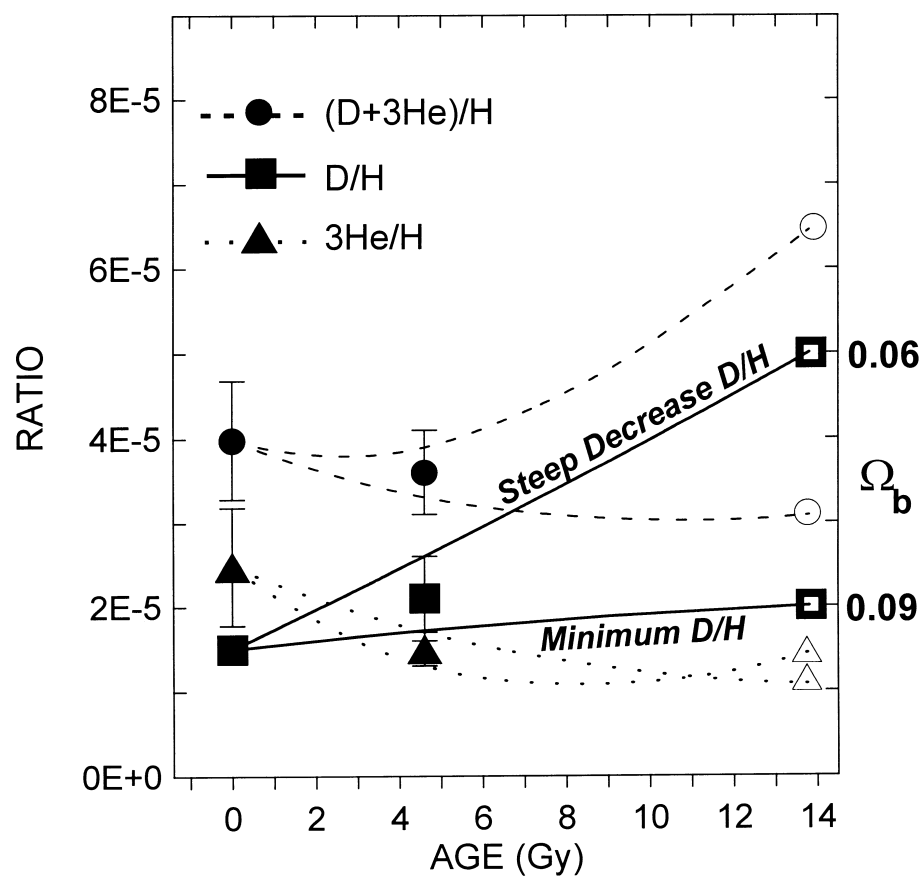

Figure 2. Illustration of the evolution of $\mathrm{D}$ and ${ }^{3} \mathrm{He}$ in the "solar ring" of the galaxy. The measured abundance data for the Local Interstellar Cloud (LIC) at age 0 are from Linsky (1998) and Gloeckler and Geiss (1998), those for the Protosolar Cloud (PSC) at age 4.6 Gy are from this work and from Mahaffy et al. (1998). The open symbols at $\sim 14$ Gy represent primordial abundances obtained from extrapolations of these galactic data. The curve labelled "Minimum D/H" leads to the minimum primordial abundance ratio inferred from the PSC data. The curve labelled "Steep Decrease D/H" leads to $(\mathrm{D} / \mathrm{H})_{\text {primordial }}=5 \times 10^{-5}$, the upper value obtained from galactic evolution studies by Tosi (1998). The dotted lines connect the measured ${ }^{3} \mathrm{He} / \mathrm{H}$ ratios with the theoretical primordial value, the dashes lines connect the measured $\left(\mathrm{D}+{ }^{3} \mathrm{He}\right) / \mathrm{H}$ ratios with the theoretical primordial $\left(\mathrm{D}+{ }^{3} \mathrm{He}\right) / \mathrm{H}$ ratio.

is higher by a factor of 2 to 10 than the baryonic density of $\Omega_{\mathrm{b}}=0.075 \pm 0.015$, the difference probably being made up of weakly interacting matter. A welcome corollary of this finding is that a dominant component of weakly interacting matter could better than baryonic matter explain the observed inhomogeneities of matter and radiation in the universe (cf. Bennett et al., 1997).

\section{Acknowledgements}

We have very much benefitted from discussions with Thomas Donahue, Hubert Reeves, Gustav Andreas Tammann, Monica Tosi, Sylvie Vauclair, Heinz Völk and Rudolf von Steiger and we thank Ursula Pfander for her help with the manuscript. This work was supported by the ISSI-Foundation, the European Space Agency and the National Aeronautics and Space Administration (NASA/JPL contract 955460). 


\section{References}

Anders, E. and Grevesse, N.: 1989, Geochim. and Cosmochim. Acta 53, 197-214.

Bahcall, J.N. and Pinsonneault, M.H.: 1995, Rev. Modern Physics 67, 781.

Bame, S.J., Hundhausen, A.J., Asbridge, J.R. and Strong, I.B.: 1968, Phys. Rev. Lett. 20, 393.

Bame, S.J., Asbridge, J.R., Feldman, W.C. and Gosling, J.T.: 1977, J. Geophys. Res. 82, 1487-1492.

Banaszkiewicz, M., Czechowski, Axford, W.I., McKenzie, J.F., and Sukhoruva, G.V.: 1998, 'Proc. 31st ESLAB Symposium', in press.

Barraclough, B.L., Feldman, W.C., Gosling, J.T., McComas, D.J., Phillips, J.L. and Goldstein, B.E.: 1996, in 'Solar Wind Eight' AIP Conf. Proc. 382, (eds. Winterhalter, D., Gosling, J.T., Habbal, S.R., Kurth, W.S., and Neugebauer, M.), AIP Press, Woodbury, N.Y., 277-280.

Bennett, L., Turner, M.S., White, M.: 1997, Physics Today November 1997, 32-38.

Black, D.C.: 1972, Geochim. Cosmochim. Acta 36, 347.

Bochsler, P.: 1984, 'Helium and Oxygen in the Solar Wind', Habilitation Thesis, University of Bern.

Bochsler, P., Geiss, J. and Maeder, A.:1990, Solar Phys. 128, 203.

Bodmer, R., Bochsler, P., Geiss, J., von Steiger, R. and Gloeckler, G.: 1995, Space Sci. Rev. 72, 61.

Bodmer, R.: 1996, 'The Helium Isotopic Ratio as a Test for Minor Ion Fractionation in the Solar Wind Acceleration Process: SWICS/ULYSSES Data Compared with Results from a Multifluid Model', Ph.D. Thesis, University of Bern.

Boltenkov, B.S., Gartmanov, V.N., Kocharov, G.E., Naidenov, V.O. and Starbunov, Ju.N.: 1972, Isvestia Akad. Nauk USSR, Ser. Phys. 34, 2319.

Bürgi, A. and Geiss, J.: 1986, Solar Phys. 103, 347.

Burles, S. and Tytler, D.: 1998, Space Sci. Rev., this volume.

Coplan, M.A., Ogilvie, K.W., Bochsler, P. and Geiss, J.: 1984, Solar Phys. 93, 415.

Eberhardt, P.: 1974, Earth Planet. Sci. Lett. 24, 182.

Encrenaz, Th. et al.: 1996, Astron. Astrophys. 315, L397-402.

Garrard, T.L. and Stone, E.C.: 1994, Adv. Space Res. 14, 589-598.

Gautier, D. and Morel, P.: 1997, Astron. Astrophys. 323, L9-L12.

Geiss, J., Hirt, P. and Leutwyler, H.: 1970, Solar Physics 12, 458.

Geiss, J., Eberhardt, P., Bühler, F., Meister, J. and Signer, P.: 1970a, J. Geophys. Res. 75, 5972.

Geiss, J. and Reeves, H.: 1972, Astron. Astrophys. 18, 126-132.

Geiss, J., Bühler, Cerutti, H., Eberhardt, P. and Filleux, Ch.: 1972 'Apollo 16 Preliminary Science Report', NASA SP-315 Section 14.

Geiss, J.: 1993, 'Origin and Evolution of the Elements' (eds. Prantzos, N., Vangioni-Flam, E., and Cassé, M.), Cambridge University Press, 89-106.

Gloeckler, G., et al.: 1997, EOS Trans. Am. Geophys. Union 78, 438.

Gloeckler, G. and Geiss, J.: 1998, Space Sci. Rev., this volume.

Grünwaldt, H.: 1976, Space Research XVI, 681-684.

Hénoux, J.-C. and Somov, B.V.: 1992, ESA SP-348, pp. 325-330.

Hollweg, J.V.: 1974, J. Geophys. Res. 79, 1539.

Kohl, J. L., et al.: 1997, 'UVCS/SOHO Empirical Determinations of anisotropic velocity distribution in the solar corona' Harvard College Observatory Preprint Series No. 4630.

Linsky, J.L.: 1998, Space Sci. Rev., this volume.

Mahaffy, P. R., et al.: 1998, Space Sci. Rev., this volume.

McKenzie, J.F., Sukhorukova, G.V. and Axford, W.I.: 1997, Astron. Astrophys., in press.

Munro, R.H. and Jackson, B.V.: 1977, Astrophys. J. 213, 874.

Niemann H.B. et al.: 1996, Science 272, 846-849.

Niemann, H.B. et al.: 1998, J. Geophys. Res., in press.

Ogilvie, K.W., Coplan, M.A., Bochsler, P. and Geiss, J.: 1980, J. Geophys. Res. 85, 6021.

Pérez Hernández, F. and Christensen-Dalsgaard, J.: 1994, MNRAS 269, 475.

Rauch, M. et al.: 1997, Astrophys. J. 489, 7.

Reeves, H. Audouze, J., Fowler, W.A. and Schramm, D.N.: 1973, Astrophys. J. 179, 909.

Schatzman, E. and Maeder, A.: 1981, Astron. Astrophys. 96, 1.

von Steiger, R. and Geiss, J.: 1989, Astron. Astrophys. 225, 222-238. 
von Steiger, R., Geiss, J. and Gloeckler, G.: 1997, in 'Cosmic Winds and the Heliosphere' (eds. Jokipii, J.R., Sonett, C.P. and Giampapa, M.S.), Tucson (Conference 1973), University of Arizona Press, pp. 581-616.

Tammann, G.A.: 1998, Space Sci. Rev., this volume.

Tosi, M.: 1998, Space Sci. Rev., this volume.

Tscharnuter, W.M.: 1987, Astron. Astrophys. 188, 55-73.

Vauclair, S.: 1998, Space Sci. Rev., this volume.

Vauclair, S.: 1998a, Space Sci. Rev., in press.

Walker, T.P., Steigman, G., Schramm, D.N., Olive, K.A. and Kang, H.S.: 1991, Astrophys. J. 376, 51.

Wieler, R., Baur, H. and Signer, P.: 1992, Lunar. Planet. Sci. XXIII, 1525-1526. 